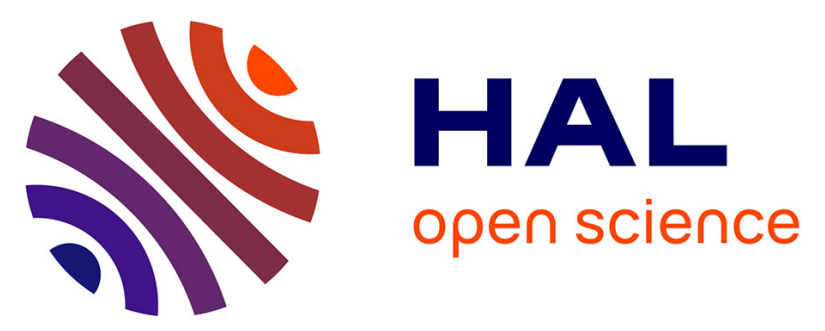

\title{
Anisotropic and hyperelastic identification of in vitro human arteries from full-field optical measurements
}

Stéphane Avril, Pierre Badel, Ambroise Duprey

\section{To cite this version:}

Stéphane Avril, Pierre Badel, Ambroise Duprey. Anisotropic and hyperelastic identification of in vitro human arteries from full-field optical measurements. Journal of Biomechanics, 2010, 43 (15), pp.2978-2985. 10.1016/j.jbiomech.2010.07.004 . hal-00543316

\section{HAL Id: hal-00543316 https://hal.science/hal-00543316}

Submitted on 6 Dec 2010

HAL is a multi-disciplinary open access archive for the deposit and dissemination of scientific research documents, whether they are published or not. The documents may come from teaching and research institutions in France or abroad, or from public or private research centers.
L'archive ouverte pluridisciplinaire HAL, est destinée au dépôt et à la diffusion de documents scientifiques de niveau recherche, publiés ou non, émanant des établissements d'enseignement et de recherche français ou étrangers, des laboratoires publics ou privés. 


\title{
Anisotropic and hyperelastic identification of in vitro human arteries from full-field optical measurements
}

\author{
Stéphane Avril, Pierre Badel, Ambroise Duprey \\ Center for Health Engineering \\ Ecole Nationale Supérieure des Mines de Saint-Étienne \\ PECM - CNRS UMR 5146 ; IFRESIS - INSERM IFR 143 \\ 158 Cours Fauriel, 42023 SAINT-ÉTIENNE cedex 2, FRANCE
}

\begin{abstract}
In this paper, we present a new approach for the bi-axial characterization of in vitro human arteries and we prove its feasibility on an example. The specificity of the approach is that it can handle heterogeneous strain and stress distributions in arterial segments. From the full-field experimental data obtained in inflation/extension tests, an inverse approach, called the virtual fields method (VFM), is used for deriving the material parameters of the tested arterial segment. The obtained results are promising and the approach can effectively provide relevant values for the anisotropic hyperelastic properties of the tested sample.
\end{abstract}

\section{${ }_{17} \quad 1 \quad$ Introduction}

It is well assessed that, despite biochemical and hemodynamical factors play a primary role in the development of most vascular disorders, solid mechanics models may contribute to understand their genesis and progression. The realism of models in solid mechanics depends significantly on the mechanical properties used as input parameters. Therefore, characterizing the biomechanical properties of arteries remains an 22 essential issue.

In vivo measurements with ultrasounds or magnetic resonance imaging (MRI) techniques provide relevant information on the vascular behavior [Slager et al., 2000, Masson et al., 2008, Avril et al., 2009] although they are not sufficient for a rigorous determination of arterial wall constitutive equations. To investigate the passive structural response of the arterial tissue, a large variety of in vitro experimental protocols are available [Humphrey, 2002].

Among them, inflation/extension of arterial segments are physiologically meaningful tests since the in vivo loading conditions may be reproduced and the native geometry is preserved [Hayashi, 1993]. Classically, for the analysis, it is assumed that the artery is a perfect cylinder and that the loading induces a homogeneous stress and strain distribution [Humphrey, 2002,Fung, 1993]. These assumptions provide a framework for deriving stress-strain curves and to fit them by appropriate constitutive models. 
However, inverse approaches should generally be employed since stresses and strains are always heterogeneous, because of noncylindrical shape [Holzapfel, 2004, Humphrey, 1999], locally varying material properties or experimental artifacts like edge effects [Arimitsu et al., 1995].

The combination of 3-D deformation measurements [Rastogi, 1999, Foster, 1978, Viotti et al., 2008, Matthys et al., 1991, Genovese, 2007, Genovese, 2009, Sutton et al., 2007] and inverse approaches [Avril et al., 2008] is now very common in solid mechanics but it is still under-employed for identifying the anisotropic hyperelastic properties of the arterial tissues [Seshaiyer and Humphrey, 2003, Einstein et al., 2005]. Moreover, the Virtual Fields Method [Grédiac et al., 2006], which is an inverse method specifically dedicated to full-field data, has never been used for the mechanical identification of arterial tissues although it has very relevant assets: insensitivity to the uncertainty of boundary conditions [Grédiac et al., 2006], robustness [Avril et al., 2004], fast convergence [Avril and Pierron, 2007].

This paper attempts to give a new contribution for addressing the mechanical identification of arterial tissues by presenting an implementation of the Virtual Fields Method to full-field experimental data measured on the whole surface of an arterial segment during inflation/extension tests. The objective of the paper is to present the principle of the approach and to prove its feasibility on an example.

\section{Materials and Methods}

\subsection{Materials}

Results reported in this paper are obtained on a fairly straight segment of a human ascending aorta (initial length: $L_{0} \simeq 35 \mathrm{~mm}$, initial average radius: $R_{0} \simeq 10 \mathrm{~mm}$, initial average thickness: $\left.e_{0} \simeq 1.3 \mathrm{~mm}\right)$. This vascular segment has been obtained from a cadaveric 65 years old female donor. All procedures were carried out in accordance with the guidelines of the Institutional Review Board of the University Hospital Centre of Saint-Etienne, France. After gently cleaning the artery with physiological solution, two side branches were clamped with surgical suture threads (Fig. 1).

\subsection{Experiments}

This study results from a collaboration between the University of Basilicata (Italy) and Ecole des Mines de Saint-Etienne (France). The experimental setup was developed at the University of Basilicata and presented in a companion paper [Genovese, 2009]. The current paper focuses on the inverse approach and all the details about the experimental techniques may be found in [Genovese, 2009].

The sample was mounted on the in vitro rig and preconditioned via 8 pressurization cycles from $p=$ 
0 to $p=80 \mathrm{mmHg}(10.5 \mathrm{kPa})$ and then 8 cycles of axial stretching from $L / L_{0}=1$ to $L / L_{0}=1.4$. Then, different sets of inflation/extension tests were performed.

Let $\boldsymbol{\Psi}_{t}$ denote the geometrical deformation of the artery induced by the loading at time $t$, such as $\mathbf{x}(t)=\mathbf{\Psi}_{t}\left(\mathbf{x}_{0}\right)$, where $\mathbf{x}(t)$ is the position vector of a material point at $t$ and $\mathbf{x}_{0}$ is the initial position vector of the same material point.

The vascular segment is covered with $N(N \simeq 300)$ black spherical markers (Fig. 1). The markers were fixed with cyanoacrylate adhesive which was shown not to diffuse in the tissues [Holzapfel et al., 2007]. The optical measurement presented in [Genovese, 2009] provides a point-wise evaluation of $\boldsymbol{\Psi}_{t}$ at all the points where a marker has been bonded, with an accuracy of $0.17 \mathrm{~mm}$.

During all the inflation/extension tests, the artery remains in a bath of physiological solution. The optical technique was calibrated accounting for the bath of physiological solution around the artery [Genovese, 2009]. However the artery was not hydrated while we bonded the markers. This lasted less than one hour. This could be reduced in the future by using glue that activates with water.

\subsection{Derivation of the field of Green-Lagrange strain tensors}

The surface of the artery is meshed for interpolating $\boldsymbol{\Psi}_{t}$ across the whole artery from the data measured at the marker position. Triangular elements with linear interpolation functions are used. The mesh is defined using a Delaunay triangularization algorithm applied onto the marker initial positions (actually the marker position means the contact position of the marker onto the surface of the artery). Each triangular element is labeled by an index denoted $q$. Let $\mathbf{x}^{q}(t)$ denote the position vector of the center of gravity of each element at time $t$

Eventually, $\boldsymbol{\Psi}_{t}$ is known everywhere across the meshed area of the artery. Let $\Omega$ be this domain across which the deformation is measured. $\Omega$ is defined in the initial configuration because the deformation is Lagrangian. Let $\omega(t)$ be the counterpart of $\Omega$ in the deformed configuration at time $t$.

All the derivations presented in this paper are based on the theory of finite deformations [Ogden, 1997]. Let $\mathbf{F}\left(\mathbf{x}_{0}, t\right)$ denote the deformation gradient:

$$
\mathbf{F}\left(\mathbf{x}_{0}, t\right) \equiv \nabla \Psi_{t}\left(\mathbf{x}_{0}\right)
$$

where $\boldsymbol{\nabla}$ is the gradient operator.

Then we define the right Cauchy-Green tensor:

$$
\mathbf{C}\left(\mathbf{x}_{0}, t\right) \equiv \mathbf{F}^{T} \mathbf{F}
$$

where $\mathbf{F}^{T}$ is the transpose of $\mathbf{F}$. 
Eventually, we define the Green-Lagrange strain tensor:

$$
\mathbf{E}\left(\mathbf{x}_{0}, t\right) \equiv[\mathbf{C}-\mathbf{I}] / 2
$$

where $\mathbf{I}$ is the identity tensor.

\subsection{Constitutive equations}

Arteries in vitro are usually considered as anisotropic visco-hyperelastic materials. Here, the viscoelastic properties are not considered and only the instantaneous and monotonic response is modeled, after preconditioning. Pseudo-elasticity can therefore be applied [Fung, 1993]. Hyperelastic models are considered.

In these models, the Cauchy stress tensor, denoted $\mathbf{S}$, is deduced from the Green-Lagrange strain tensor such as:

$$
\mathbf{S}=\mathbf{F}: d \Phi / d \mathbf{E}: \mathbf{F}^{T}+\lambda \mathbf{I}
$$

where $\Phi$ is the strain energy function depending only on $\mathbf{E}$ and on material parameters. The term $\lambda \mathbf{I}$ comes from the incompressibility and $\lambda$ is assessed by writing the plane stress condition $\left(S_{33}=0\right)$.

Different strain energy functions for the anisotropic hyperelastic behavior of arteries may be found in the literature. Some of them are phenomenological [Fung, 1993, Sun and Sacks, 2005] and others are based on the microstructure [Holzapfel et al., 2000]. In this study, we consider 3 different models:

1. the Delfino model [Holzapfel et al., 2000]:

$$
\Phi^{\prime}=\frac{1}{2} \beta^{\prime}\left[\exp \left(\alpha^{\prime}\left(I_{1}-3\right)\right)-1\right]
$$

where $\beta^{\prime}$ and $\alpha^{\prime}$ are material parameters and $I_{1}=\operatorname{tr}(\mathbf{C})$.

2. the 2-D Fung model [Fung, 1993], which may be written as:

$$
\Phi=\frac{1}{2} \beta\left[\exp \left(\alpha_{11}\left(E_{11}\right)^{2}+\alpha_{22}\left(E_{22}\right)^{2}+\alpha_{12} E_{11} E_{22}\right)-1\right]=\frac{1}{2} \beta[\exp (Q)-1]
$$

where $\beta, \alpha_{11}, \alpha_{22}$ and $\alpha_{12}$ are material parameters and $E_{i j}$ are the components of the $\mathbf{E}$ matrix in the material coordinate system (indice 1 refers to the circumferential direction and indice 2 refers to the axial direction in the local coordinate system). 
3. the Holzapfel model [Holzapfel et al., 2000]. This model assigns separate strain energy functions to the media layer and the adventitia layer. The simplest form of the strain energy function may be written:

$$
\Phi^{\prime \prime}=\frac{c^{\prime \prime}}{2}\left(I_{1}-1\right)+\frac{k_{1}^{\prime \prime}}{2 k_{2}^{\prime \prime}}\left[\exp \left(k_{2}^{\prime \prime}\left(I_{4}-1\right)^{2}\right)-1\right]+\frac{k_{1}^{\prime \prime}}{2 k_{2}^{\prime \prime}}\left[\exp \left(k_{2}^{\prime \prime}\left(I_{6}-1\right)^{2}\right)-1\right]
$$

where $c^{\prime \prime}$ and $k_{1}^{\prime \prime}$ are stress-like material parameters, $k_{2}^{\prime \prime}$ is a dimensionless material parameter; $I_{4}$ and $I_{6}$ are the squares of the stretch component in the two families of symmetric tissue fibers. Hence, this model prescribes a fiber orientation angle $\phi$ for each layer, based usually on analysis of microscopy data. The fiber angles for the specimen used in this work were not known, and so the fiber angles are another material parameter to be determined from the experimental data.

\subsection{Inverse approach}

The Virtual Fields Method (VFM) is an approach dedicated to the identification of material parameters from full-field measurements [Grédiac et al., 2006, Avril et al., 2004, Avril and Pierron, 2007, Promma et al., 2009]. It is based on the weak form of equilibrium equations.

Assuming quasi-static conditions and no body forces, the weak form of equilibrium in the deformed configuration may be written such as:

$$
-\int_{\omega(t)} \mathbf{S}: \mathbf{E}^{*} d v+\int_{\partial \omega(t)} \mathbf{t} \cdot \mathbf{u}^{*} d s=0
$$

where $\partial \omega(t)$ is the boundary of the volume in the deformed configuration, vector $\mathbf{t}$ denotes the tractions applied onto this boundary (pressure forces and axial load), $\mathbf{u}^{*}$ is a displacement virtual field which acts as a test function in Eq. 5). $\mathbf{E}^{*}$ is the strain virtual field deduced from the gradient of $\mathbf{u}^{*}$ :

$$
\mathbf{E}^{*}=\frac{1}{2}\left(\nabla \mathbf{u}^{*}+\nabla^{T} \mathbf{u}^{*}\right)
$$

At position $\mathbf{x}$ where a marker is located, $\mathbf{u}^{* 1}$ and $\mathbf{u}^{* 2}$ take the following values:

$$
\left\{\begin{array}{l}
\mathbf{u}^{* 1}=\sin \left(\pi \frac{z-z_{\mathrm{b}}}{z_{\mathrm{t}}-z_{\mathrm{b}}}\right) \mathbf{i} \\
\mathbf{u}^{* 2}=\frac{z-z_{\mathrm{t}}}{z_{\mathrm{b}}-z_{\mathrm{t}}} \mathbf{k}
\end{array}\right.
$$

where: 
- vectors $\mathbf{i}$ and $\mathbf{k}$ are respectively the radial and longitudinal axes in the global cylindrical coordinate system defined in Fig. 2;

- $z$ is the component of $\mathbf{x}$ along the longitudinal axis: $z=\mathbf{x} \cdot \mathbf{k}$;

- $z_{\mathrm{b}}$ and $z_{\mathrm{t}}$ are defined in Fig. 2 .

The rationale behind this choice of virtual displacements is:

- one involves mainly the radial properties $\left(\mathbf{u}^{* 1}\right)$

- one involves mainly the axial properties $\left(\mathbf{u}^{* 2}\right)$

- both zero the action of unknown reaction forces or involve their resultant if the resultant is measured [Grédiac et al., 2006].

Given the previous points, infinity of virtual deformation may be used. The rationale behind the current choice is that the best results are always obtained with the simplest functions. This was proved in different papers [Grédiac et al., 2006, Avril et al., 2004, Avril and Pierron, 2007, Promma et al., 2009]. This can be interpreted like this: a virtual deformation is a test function. If the test function is very smooth, it will average out the randomly distributed errors contained in the experimental data.

Eq. 7 only gives the values of the virtual displacement at marker positions. Between the marker positions, the values of $\mathbf{u}^{* 1}$ and $\mathbf{u}^{* 2}$ are interpolated linearly.

Moreover, regarding the external virtual work, it can be written:

$$
\left\{\begin{array}{l}
\int_{\partial \omega(t)} \mathbf{t} \cdot \mathbf{u}^{* 1} d s=\int_{\partial \omega(t)} \mathbf{k}_{t} \cdot \mathbf{u}^{* 1} p(t) d s \\
\int_{\partial \omega(t)} \mathbf{t} \cdot \mathbf{u}^{* 2} d s=\int_{\partial \omega(t)} \mathbf{k}_{t} \cdot \mathbf{u}^{* 2} p(t) d s+\mathcal{F}(t)
\end{array}\right.
$$

where $\mathbf{k}_{t}$ is the unit outer normal vector at $t, \mathcal{F}(t)$ is the axial resultant load measured by a load cell.

As $\mathbf{S}$ is deduced from experimental data, Eq. 5 cannot be satisfied exactly and the principle of the inverse method [Grédiac et al., 2006] is to minimize the squares of residuals, as defined in the following cost function:

$$
\zeta\left(\beta, \alpha_{11}, \alpha_{22}, \alpha_{12}\right)=\sum_{k} \sum_{m}\left[-\int_{\omega\left(t_{m}\right)} \mathbf{S}: \mathbf{E}^{* k} d v+p\left(t_{m}\right) \int_{\partial \omega\left(t_{m}\right)} \mathbf{k}_{t_{m}} \cdot \mathbf{u}^{* k} d s+\delta_{k 2} \mathcal{F}(t)\right]^{2}
$$


where $m$ labels the time when a measurement is achieved and $k$ labels the virtual field $(k=1,2) ; \delta_{k 2}=0$ if $k=1$ and $\delta_{k 2}=1$ if $k=2$.

Considering the interpolation of all quantities with the triangular mesh, the previous integrals in the principle of virtual work may be changed into discrete sums.

$$
\begin{aligned}
& \zeta\left(\beta, \alpha_{11}, \alpha_{22}, \alpha_{12}\right)= \\
& \sum_{k} \sum_{m}\left[-\sum_{q} \mathbf{S}\left(\mathbf{x}^{q, m}\right): \mathbf{E}^{* k}\left(\mathbf{x}^{q, m}\right) A\left(\mathbf{x}^{q, m}\right) e\left(\mathbf{x}^{q, m}\right)+p\left(t_{m}\right) \sum_{q} \mathbf{k}_{t}\left(\mathbf{x}^{q, m}\right) \cdot \mathbf{u}^{* k}\left(\mathbf{x}^{q, m}\right) A\left(\mathbf{x}^{q, m}\right)+\delta_{k 2} \mathcal{F}(t)\right]^{2}
\end{aligned}
$$

where $A\left(\mathbf{x}^{q, m}\right)$ denotes the area of triangular element $q$ at time $t_{m}$ and $e\left(\mathbf{x}^{q, m}\right)$ denotes the thickness of the artery ${ }^{1}$ at $\mathbf{x}^{q}$ at time $t_{m} . \mathbf{E}^{* k}$ is derived from the virtual displacements $\mathbf{u}^{* k}$ using Eq. 6. The virtual displacements $\mathbf{u}^{* k}$ are defined in Eq. 7. The value of $p$ are experimental data and $\mathbf{S}$ is derived using Eq. 1. It must be noted that the model parameters are involved in $\mathbf{S}$. All the incremental steps are taken into account as the squares of residuals of each incremental step, denoted $m$, are summed up.

Cost function $\zeta$ figures the quadratic gap between the internal virtual work (IVW) and the external virtual work (EVW) where:

$$
\begin{aligned}
& \mathrm{IVW}^{k, m}=e_{0} \sum_{q} \mathbf{S}\left(\mathbf{x}^{q, m}\right): \mathbf{E}^{* k}\left(\mathbf{x}^{q, m}\right) A\left(\mathbf{x}^{q, 0}\right) \\
& \mathrm{EVW}^{k, m}=p\left(t_{m}\right) \sum_{q} \mathbf{k}_{t}\left(\mathbf{x}^{q, m}\right) \cdot \mathbf{u}^{* k}\left(\mathbf{x}^{q, m}\right) A\left(\mathbf{x}^{q, m}\right)+\delta_{k 2} \mathcal{F}(t)
\end{aligned}
$$

Cost function $\zeta$ and IVW are driven by the choice of the unknown material parameters. Eventually, the cost function can be minimized through an iterative scheme using the Nelder-Mead algorithm [Nelder and Mead, 1965]. This yields the unknown material parameters.

\section{Results}

A sample was tested for proving the feasibility of the approach (Fig. 1 and Fig. 2). We report data corresponding to 8 pressure levels distributed between 0 and $150 \mathrm{mmHg}$ at $L / L_{0}=1.1$ (Fig. 3 and Fig. 4).

The results obtained with the VFM are reported in Tab. 1 for the Fung model. Convergence of the Nelder Mead optimization routine was reached in nearly 10 minutes. The identified values for the Fung material parameters of the artery are consistent with the range orders reported in the literature [Holzapfel

\footnotetext{
${ }^{1}$ Due to the incompressibility assumption, $A\left(\mathbf{x}^{q, m}\right) e\left(\mathbf{x}^{q, m}\right)=A\left(\mathbf{x}^{q, 0}\right) e_{0}$, with $\mathbf{x}^{q, m}=\mathbf{x}^{q}\left(t_{m}\right)$.
} 


\begin{tabular}{|c|c|c|c|c|}
\hline parameter & $\beta$ & $\alpha_{11}$ & $\alpha_{22}$ & $\alpha_{12}$ \\
\hline identified value & $5 \mathrm{kPa}$ & 14.5 & 7 & 0.1 \\
\hline
\end{tabular}

Table 1: Results obtained with experimental data for the Fung model.

et al., 2000, Fung, 1993]. Parameter $\beta$ is usually around $10 \mathrm{kPa}$. It is interesting to notice the large difference between the value of $\alpha_{11}$ and $\alpha_{22}$. It means that there is a large anisotropy in this specimen, which has already been reported in the literature [Holzapfel et al., 2000].

It can be observed in Fig. 5 that the gap between the external virtual work and the internal virtual work is very low for the obtained material parameters.

\section{Discussion}

\subsection{Application to the other constitutive models}

\subsubsection{The Delfino model}

The material parameters of Eq. 2, namely $\alpha^{\prime}$ and $\beta^{\prime}$ can be identified using only $\mathbf{u}^{* 1}$. This means that only the circumferential response is used for the identification. The results obtained with the VFM for these two material parameters are: $\beta^{\prime}=1.5 \mathrm{kPa}$ and $\alpha^{\prime}=4.6$.

However, it is interesting to notice in Fig. 6 that the gap between IVW and EVW remains very large for $\mathbf{u}^{* 2}$ when computed with the constitutive equations of Eq. 2 and the material parameters reported above. This indicates irrelevancy of the isotropic assumption. This justifies the choice of anisotropic laws.

\subsubsection{The Holzapfel model}

Here, having access only to measurements on the surface of the artery, it is only possible to identify the average strain energy function across the whole thickness, but not separately strain energy functions of the media layer and of the adventitia layer. If, as in [Marra et al., 2006], the strain energy function is assumed homogeneous across the thickness, then a mean fiber angle and mean values of the other parameters of the Holzapfel model can be identified by the VFM. The obtained results are: $c^{\prime \prime}=0.4 \mathrm{kPa}, k_{1}^{\prime \prime}=8.7 \mathrm{kPa}$, $k_{2}^{\prime \prime}=5.4$ and $\phi=27^{\circ}$. These results show that parameters of a Holzapfel model may be retrieved by the VFM.

Fiber angles and material parameters in human arteries are usually different for the media and the adventitia. Only average values are reported here. A separate identification of media and adventitia constitutive equations would be possible by mechanical separation of the layers of the human artery, as 
shown in [Holzapfel et al., 2000].

\subsubsection{Material heterogeneities}

Arteries may also have properties varying along the axis. The VFM was already applied successfully to the identification of nonlinear heterogeneous behavior for metals [Sutton et al., 2008]. The implementation of a similar approach for arteries is possible but it will require that the spatial resolution is adapted to the length scale of local variations of the material properties. This may be achieved by employing 3-D digital image correlation, as shown in [Sutton et al., 2007].

\subsection{Discussions for $\alpha_{12}$ in the Fung model}

Parameter $\alpha_{12}$ is the coupling factor between $E_{11}$ and $E_{22}$ in the exponential of the strain energy function. It means that $\alpha_{12}$ characterizes the effect of the circumferential strain onto the axial response, or viceversa. A value of 0.1 was found for parameter $\alpha_{12}$ (Tab. 1). For obtaining this result, the algorithm was initialized according to values ${ }^{2}$ reported in [Fung, 1993].

However, it must be noted that the results regarding $\alpha_{12}$ are significantly affected by the value input for initializing the optimization algorithm. This may indicate a lack of sensitivity of the cost function to $\alpha_{12}$, inducing the existence of a valley in the cost function.

The dependence of the VFM to the initializing values was not observed for the Holzapfel model.

Nevertheless, in the Holzapfel model, the exponential part is driven by only 3 material parameters, whereas the exponential part of the Fung model is driven by 4 material parameters. This supplementary material parameter in the Fung model does not affect sufficiently the value of cost function $\zeta$ in Eq. 10, inducing the lack of sensitivity to $\alpha_{12}$. If one would like to increase the sensitivity to $\alpha_{12}$, one would have to consider the response of the artery not only to incrementally varying pressures, but also to incrementally varying stretches in the axial direction, and to include these responses in the definition of cost function $\zeta$. This will be considered in future studies.

\subsection{Implementation of the identified model in a FE code}

In [Sun and Sacks, 2005], the implementation of the Fung model in a FE code was discussed. In order to check the feasibility of utilizing this model with the parameters that we identified here (Tab. 1), computations were achieved on the geometry of the artery with the Abaqus ${ }^{\circledR}$ software. The geometry of the model is a $33.8 \mathrm{~mm}$ long cylinder with an initial diameter of $21.5 \mathrm{~mm}$, and initial thickness of $1.3 \mathrm{~mm}$.

\footnotetext{
${ }^{2}$ Initial values: $\beta=29 \mathrm{kPa}, \alpha_{11}=2.5, \alpha_{22}=0.5$ and $\alpha_{12}=0.17$
} 
It was meshed with 1276 membrane elements (M3D4R type in Abaqus $\left.{ }^{\circledR}\right)$. The Fung model is a built-in feature of Abaqus ${ }^{\circledR}$, which requires the definition of a local coordinate system related to the anisotropy directions and the constitutive parameters reported in Tab. 1. No residual stress was incorporated in this model. One end of the cylinder was clamped. Regarding the other end, radial displacements were fixed and a longitudinal displacement was prescribed, corresponding to $L / L_{0}=1.1$. The different experimental pressure steps were applied successively. The resolution of the problem was performed using an implicit scheme accounting for large strains.

A comparison of the average radii computed by Abaqus ${ }^{\circledR}$ to the measured average radii is shown in Fig. 7. The comparison is made for the cross section located at $z=\left(z_{\mathrm{b}}+z_{\mathrm{t}}\right) / 2$, i.e. at midspan. The results show a good agreement.

However, the radii computed by FE model are slightly larger than the experimental ones. It may be induced by the fact that, in the model, we neglected the pressure applied by the physiological bath on the external surface of the artery (about $5 \mathrm{mmHg} \simeq 0.7 \mathrm{kPa}$ at the midspan of the arterial segment). Discrepancies may also be induced by a difference between the experimental boundary conditions and the actual ones at the ends. For the FE model, the boundary conditions were of clamping type at both ends, whereas the actual ones are less rigid.

It is worth noting here that this discussion about the boundary conditions at both ends does not affect the results obtained by the VFM. Indeed, the effect of unknown reaction forces and parasitic motions at both ends is filtered out by the VFM. This is an essential asset of the VFM, as shown in [Grédiac et al., 2006].

\subsection{Derivation of average stress/strain curves}

It was shown that the identified Fung model can be used to derive stress/strain curves of the artery, both in the circumferential direction and in the axial direction. Results are presented in the appendices.

\section{Conclusion}

In conclusion, results presented in this work are promising regarding the application of the virtual fields method (VFM) for identifying the anisotropic hyperelastic properties of arteries. An innovative experimental device has been set up, calibrated and validated for characterizing arterial mechanical properties.

It is now important to carry out a large number of experiments for validating the approach with different sets of data and different tests. Other perspectives concern more complex loading conditions 
and more sophisticated models. More especially, next steps will consist in (i) considering the possible heterogeneity of the properties and geometry of the artery wall, (ii) processing data from different loading cases (including torsion), (iii) and considering independently the different layers of arteries and the residual stresses. Achievement of these steps will require optimal spatial resolution and accuracy of the measurement technique, employing for instance 3-D digital image correlation [Sutton et al., 2007].

\section{Acknowledgements}

The authors would like to thank Professeur Jean-Pierre Favre and to his staff in the department of cardiovascular surgery at the University Hospital of Saint-Etienne (France), for their help in preparing the specimen tested in this study. The authors are also very grateful to Dr. Katia Genovese for the experimental work. This study is part of the Imandef project (Grant ANR-08-JCJC-0071) funded by the ANR (French National Research Agency).

\section{Appendices}

\subsection{Appendix A}

In this section, we show that the identified Fung model can be used to derive a stress/strain curve of the artery in the circumferential direction and that this curve is in agreement with the average stress/strain curve deduced from experimental data.

The average circumferential Green-Lagrange strain is defined by:

$$
\tilde{E}_{11}(t)=\frac{1}{2}\left(\left(\frac{\bar{R}}{R_{0}}\right)^{2}-1\right)
$$

where $\bar{R}(t)$ is the average radius of the best fitting circle at time $t$ at $x_{3}=\left(x_{3}^{\mathrm{b}}+x_{3}^{\mathrm{t}}\right) / 2$ and $R_{0}$ is $\bar{R}(t)$ at $t=0$

The circumferential stress component, denoted $\tilde{S}_{11}$, should satisfy the Laplace law everywhere:

$$
\tilde{S}_{11}(t)=\frac{p(t) \bar{R}(t)}{\bar{e}(t)}
$$

where $\bar{e}(t)$ is the average thickness at the midspan of the arterial segment.

We compare the value of $\tilde{S}_{11}(t)$ and $\bar{S}_{11}(t)$, where $\bar{S}_{11}(t)$ is the value deduced from $\tilde{E}_{11}$ by applying directly the Fung model of Eq. 3 to $\tilde{E}_{11}$ with the assumption $\tilde{E}_{22}=0$. The parameters for the Fung's model are the ones that are reported above in Tab. 1. 
Results shown in Fig. 8a are in agreement. This means that the material parameters identified in the circumferential direction are consistent with a standard procedure (plotting stress/strain curves from pressure/diameter measurements). The advantage of the VFM is that the properties in the axial direction were also identified simultaneously.

\subsection{Appendix B}

In this appendix, we show that the identified Fung model can be used to derive a stress/strain curve of the artery subjected to a tensile test in the axial direction and that this curve is in agreement with the average stress/strain curve deduced from experimental data.

The axial Green-Lagrange strain is defined by:

$$
\tilde{E}_{22}(t)=\frac{1}{2}\left(\left(\frac{L}{L_{0}}\right)^{2}-1\right)
$$

where $L(t)$ is the length of the arterial segment at time $t$.

The axial stress component, denoted $\tilde{S}_{22}$ should satisfy:

$$
\tilde{S}_{22}(t)=\frac{\mathcal{F}(t)}{2 \pi \bar{R}(t) \bar{e}(t)}
$$

We compare the value of $\tilde{S}_{22}(t)$ and $\bar{S}_{22}(t)$, where $\bar{S}_{22}(t)$ is the value deduced from $\tilde{E}_{22}$ by applying directly the Fung model of Eq. 3 to $\tilde{E}_{22}$ with the assumption $\tilde{E}_{11}=0$. The parameters for the Fung's model are reported in Tab. 1.

Results shown in Fig. 8b are in agreement. This means that the material parameters identified in the axial direction are in agreement with a classical analysis consisting in plotting stress/strain curves. The advantage of the VFM is that the properties in the axial direction were identified using only one stretch value, whereas a full tensile test was required in the axial direction for plotting the stress/strain curve with the standard analysis.

\section{References}

[Arimitsu et al., 1995] Arimitsu, Y., Nishioka, K., , and Senda, T. (1995). A study of Saint-Venant's principle for composite materials by means of internal stress fields. Journal of Applied Mechanics, 62:53-62. 
[Avril et al., 2008] Avril, S., Bonnet, M., Bretelle, A.-S., Grédiac, M., Hild, F., Ienny, P., Latourte, F., Lemosse, D., Pagano, S., Pagnacco, E., and Pierron, F. (2008). Identification from measurements of mechanical fields. Experimental Mechanics, 48(5):381-402.

[Avril et al., 2004] Avril, S., Grédiac, M., and Pierron, F. (2004). Sensitivity of the virtual fields method to noisy data. Computational Mechanics, 34(6):439-452.

[Avril et al., 2009] Avril, S., Huntley, J., and Cusack, R. (2009). In-vivo measurements of blood viscosity and wall stiffness in the carotid using PC-MRI. European Journal of Computational Mechanics, 18(1):920 .

[Avril and Pierron, 2007] Avril, S. and Pierron, F. (2007). General framework for the identification of constitutive parameters from full-field measurements in linear elasticity. International Journal of Solids and Structures, 44:4978-5002.

[Einstein et al., 2005] Einstein, D., Freed, A., Stander, N., Fata, B., and Vesely, I. (2005). Inverse parameter fitting of biological tissues: A response surface approach. Annals of Biomedical Engineering, 33(12):1819-1830.

[Foster, 1978] Foster, C. (1978). Measurement of radial deformations in thin-walled cylinders. Experimental Mechanics, 18:426-430.

[Fung, 1993] Fung, Y. (1993). Biomechanics: Mechanical Properties of Living Tissues. New York: Springer.

[Genovese, 2007] Genovese, K. (2007). Radial metrology application to whole-body measurement on hyperelastic tubular samples. Optics and Lasers in Engineering, 45(11):1059-1066.

[Genovese, 2009] Genovese, K. (2009). A video-optical system for time-resolved whole-body measurement on vascular segments. Optics and Lasers in Engineering, 47:995-1008.

[Grédiac et al., 2006] Grédiac, M., Pierron, F., Avril, S., and Toussaint, E. (2006). The virtual fields method for extracting constitutive parameters from full-field measurements: a review. Strain, 42:233253.

[Hayashi, 1993] Hayashi, K. (1993). Experimental approaches on measuring the mechanical properties and constitutive laws of arterial walls. ASME Journal of Biomechical Engineering, 115:481-488. 
[Holzapfel, 2004] Holzapfel, G. (2004). Experimental approaches on measuring the mechanical properties and constitutive laws of arterial walls. Encyclopedia of computational mechanics. Solids and structures, 2:605-635

[Holzapfel et al., 2000] Holzapfel, G., Gasser, T., and Ogden, R. (2000). A new constitutive framework for arterial wall mechanics and comparative study of material models. Journal of Elasticity, 61:1-48.

[Holzapfel et al., 2007] Holzapfel, G., Sommer, G., Auer, M., Regitnig, P., and Ogden, R. (2007). Layerspecific $3 \mathrm{~d}$ residual deformations of human aortas with non-atherosclerotic intimal thickening. Annals of Biomedical Engineering, 35(4):530-545.

[Humphrey, 1999] Humphrey, J. (1999). An evaluation of pseudoelastic descriptors used in arterial mechanics. ASME Journal of Biomechical Engineering, 121:259-262.

[Humphrey, 2002] Humphrey, J. (2002). Cardiovascular solid mechanics - Cells, tissues and organs. New York: Springer.

[Marra et al., 2006] Marra, S., Kennedy, F., Kinkaid, J., and Fillinger, M. (2006). Elastic and rupture properties of porcine aortic tissue measured using inflation testing. Cardiovascular Engineering, 6:125133.

[Masson et al., 2008] Masson, I., Boutouyrie, P., Laurent, S., Humphrey, J., and Zidi, M. (2008). Characterization of arterial wall mechanical behavior and stresses from human clinical data. Journal of Biomechanics, 41(12):2618-2627.

[Matthys et al., 1991] Matthys, D., Gilbert, J., and Greguss, P. (1991). Endoscopic measurement using radial metrology with digital correlation. Optical Engineering, 30(19):1400-1455.

[Nelder and Mead, 1965] Nelder, J. and Mead, R. (1965). A simplex method for function minimization. Computer Journal, 7(4):308-313.

[Ogden, 1997] Ogden, R. (1997). Non-linear Elastic Deformations. Dover Publication, New York.

[Promma et al., 2009] Promma, N., Raka, B., Grédiac, M., Toussaint, E., Cam, J. L., Balandraud, X., and Hild, F. (2009). Application of the virtual fields method to mechanical characterization of elastomeric materials. International Journal of Solids and Structures, 46:698-715.

[Rastogi, 1999] Rastogi, P. (1999). Photomechanics. Springer Verlag. 
[Seshaiyer and Humphrey, 2003] Seshaiyer, P. and Humphrey, J. (2003). A sub-domain inverse finite element characterization of hyperelastic membranes including soft tissues. Journal of Biomechanical Engineering, 125:363-371.

[Slager et al., 2000] Slager, C., Wentzel, J., Schuurbiers, J., Oomen, J., Kloet, J., Krams, R., von Birgelen, C., van der Giessen, W., Serruys, P., and de Feyter, P. (2000). True 3-dimensional reconstruction of coronary arteries in patients by fusion of angiography and IVUS and its quantitative validation. Circulation, 102:511-516.

[Sun and Sacks, 2005] Sun, W. and Sacks, M. (2005). Finite element implementation of a generalized Fung-elastic constitutive model for planar soft tissues. Biomechanics and Modelling in Mechanobiology, 4:190-199.

[Sutton et al., 2007] Sutton, M., Ke, X., Lessner, S., Goldbach, M., Yost, M., Zhao, F., and Schreier, H. (2007). Strain field measurements on mouse carotid arteries using microscopic three-dimensional digital image correlation. Journal of Biomedical Materials Research Part A, 84A(1):178-190.

[Sutton et al., 2008] Sutton, M., Yan, J., Avril, S., Pierron, F., and Adeeb, S. (2008). Identification of heterogeneous constitutive parameters in a welded specimen: Uniform stress and virtual fields methods for material property estimation. Experimental Mechanics, 48(5):451-464.

[Viotti et al., 2008] Viotti, M., Albertazzi, A., Fantin, A., and Pont, A. D. (2008). Comparison between a white-light interferometer and a tactile formtester for the measurement of long inner cylindrical surfaces. Optics and Lasers in Engineering, 46:396-403. 


\section{List of Figures}

1 Picture of the arterial segment used in the tests. . . . . . . . . . . . . . . . . 17

2 Meshing and schematic of the initial geometry: (a) 3D view (b) cross sectional view. . . . 18

3 Plot of the circumferential components of the Green-Lagrange strain tensor for different values of $p$. The plot is displayed in the undeformed configuration. . . . . . . . . . . . . 19

4 Plot of the circumferential components of the Cauchy stress tensor for different values of p. The plot is displayed in the deformed configuration. The Cauchy stress are calculated with the Fung model using the identified values reported in Tab.1 . . . . . . . . . . . . . . 20

5 Comparison of the internal and external virtual work for virtual field 1 (a) and virtual field 2 (b) when an anisotropic model is identitied . . . . . . . . . . . . . . . . . . . 21

$6 \quad$ Comparison of the internal and external virtual work for virtual field 1 (a) and virtual field 2 (b) when an isotropic model is identitied . . . . . . . . . . . . . . . . . . 22

7 Comparison of the deformed geometry between a FE model and experimental data . . . . 23

8 Obtained stress/strain curves: (a) in the circumferential direction and (b) in the axial

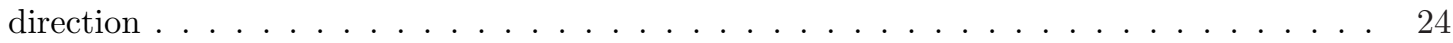



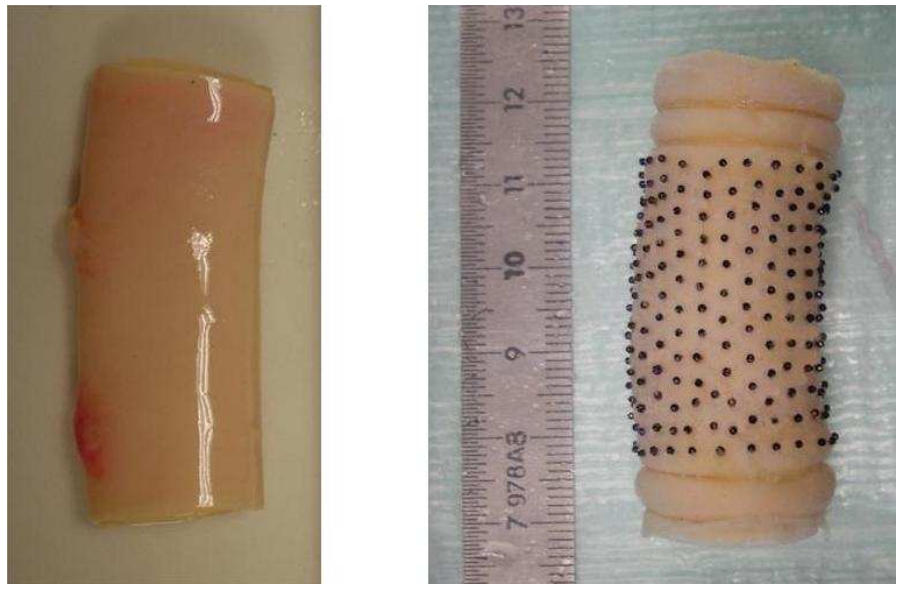

Figure 1: Picture of the arterial segment used in the tests. 


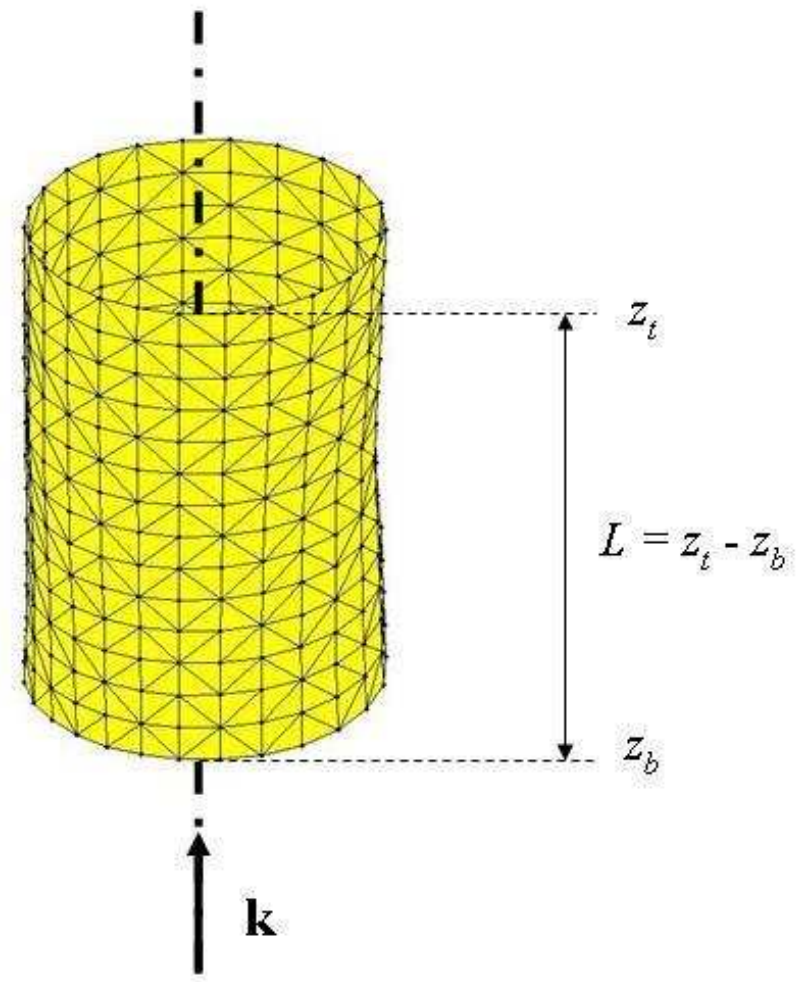

(a)

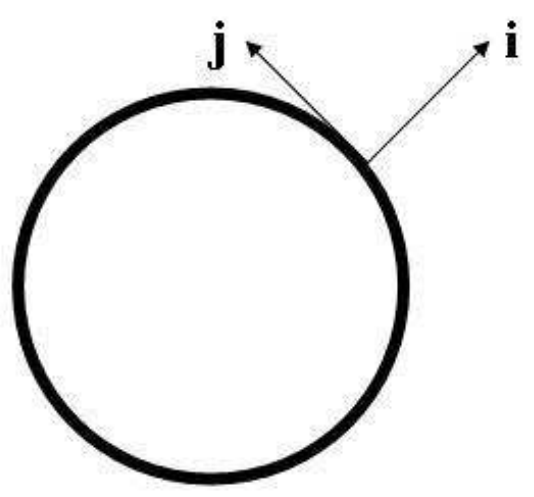

(b)

Figure 2: Meshing and schematic of the initial geometry: (a) 3D view (b) cross sectional view. 

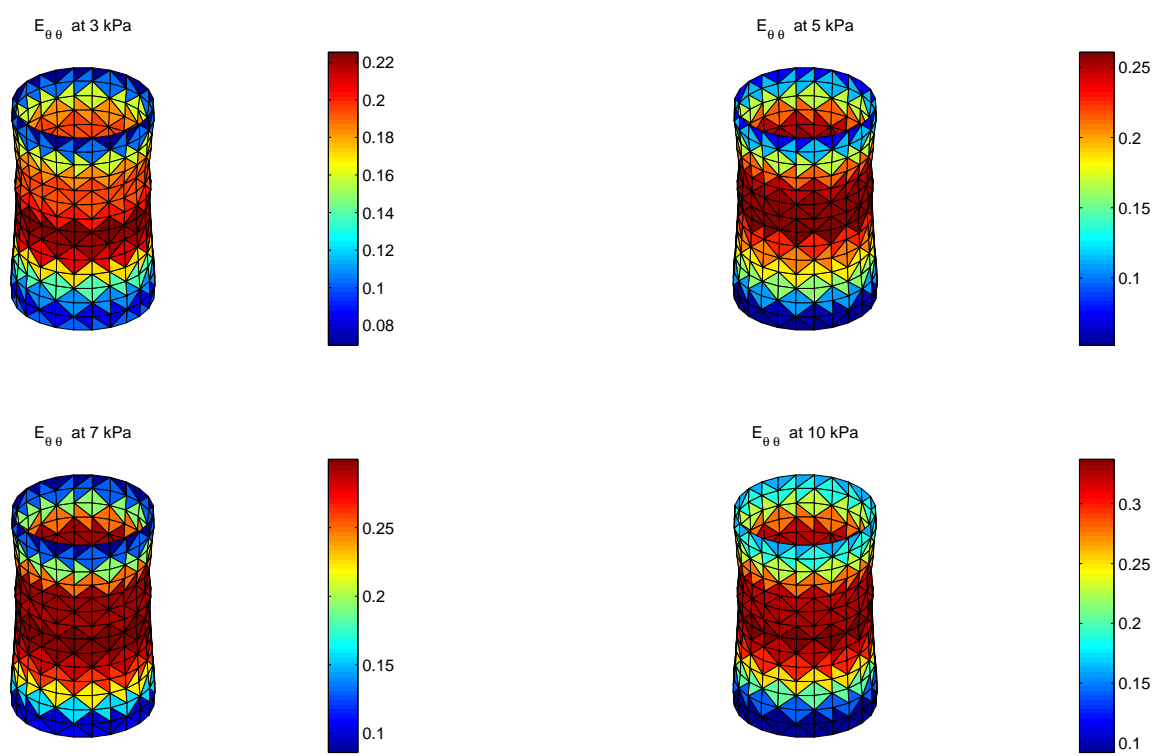

Figure 3: Plot of the circumferential components of the Green-Lagrange strain tensor for different values of $p$. The plot is displayed in the undeformed configuration. 

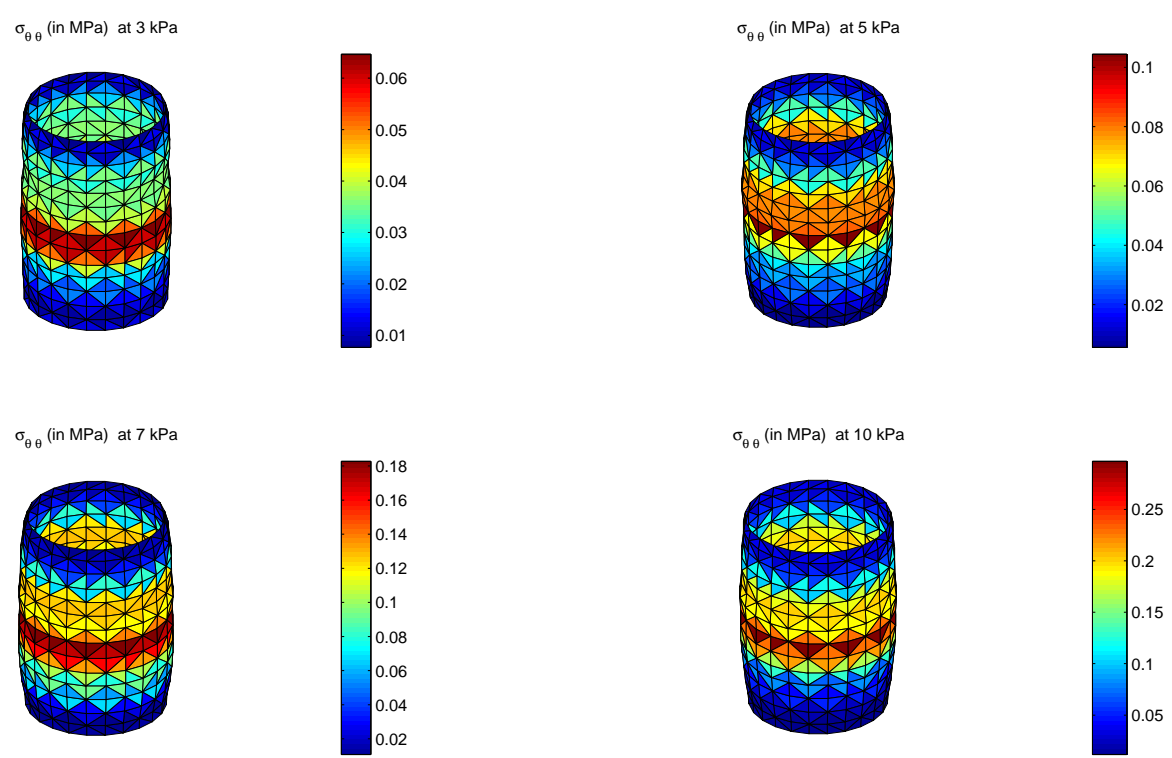

Figure 4: Plot of the circumferential components of the Cauchy stress tensor for different values of $p$. The plot is displayed in the deformed configuration. The Cauchy stress are calculated with the Fung model using the identified values reported in Tab.1 

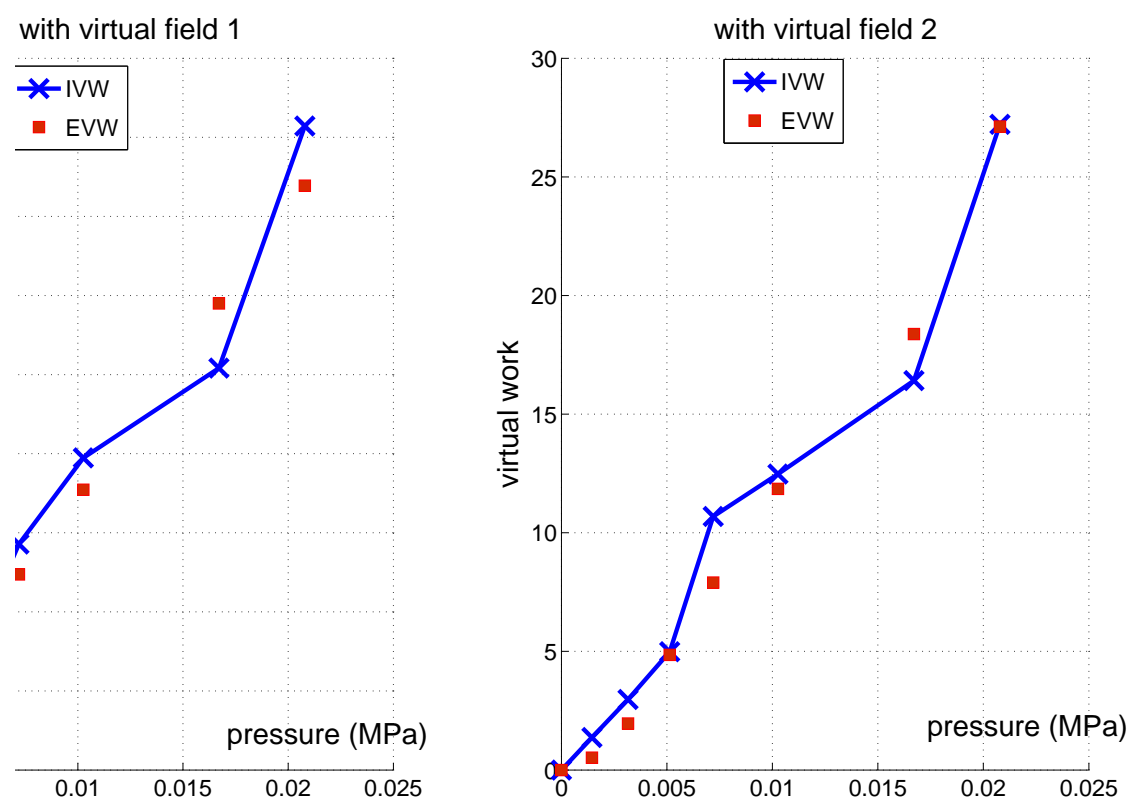

Figure 5: Comparison of the internal and external virtual work for virtual field 1 (a) and virtual field 2 (b) when an anisotropic model is identitied 

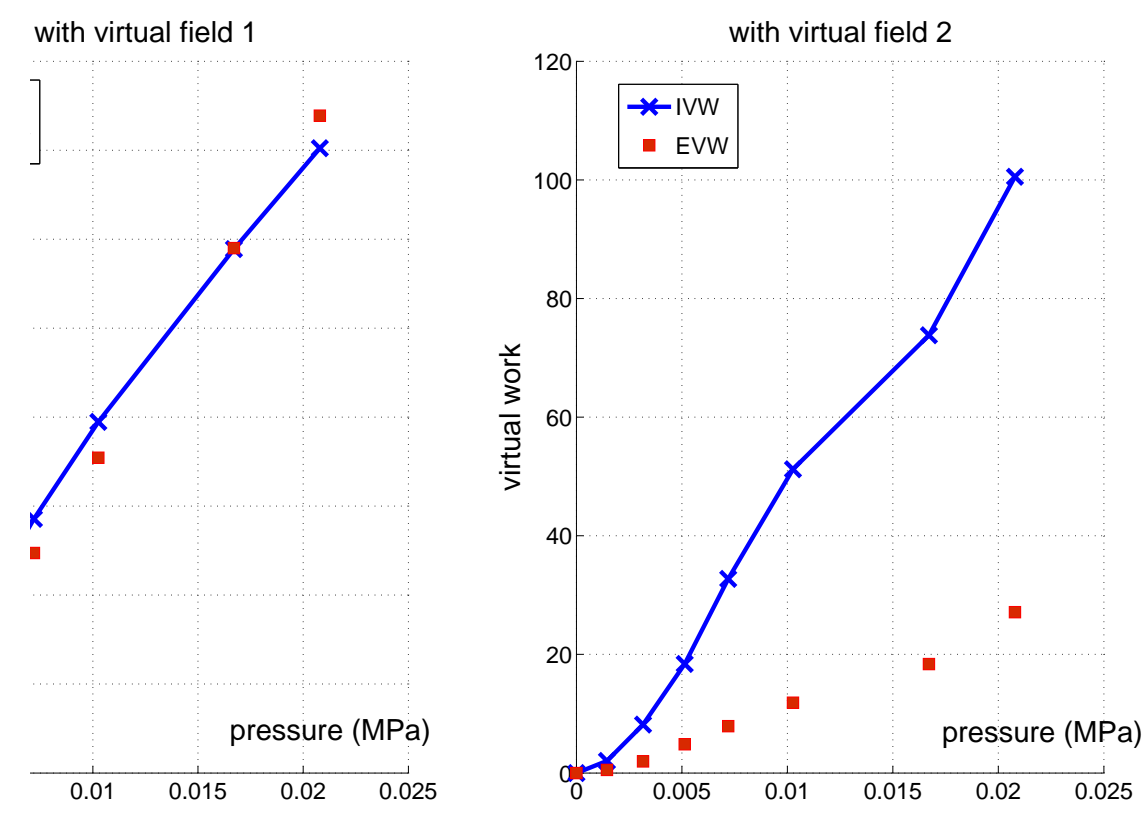

Figure 6: Comparison of the internal and external virtual work for virtual field 1 (a) and virtual field 2 (b) when an isotropic model is identitied 


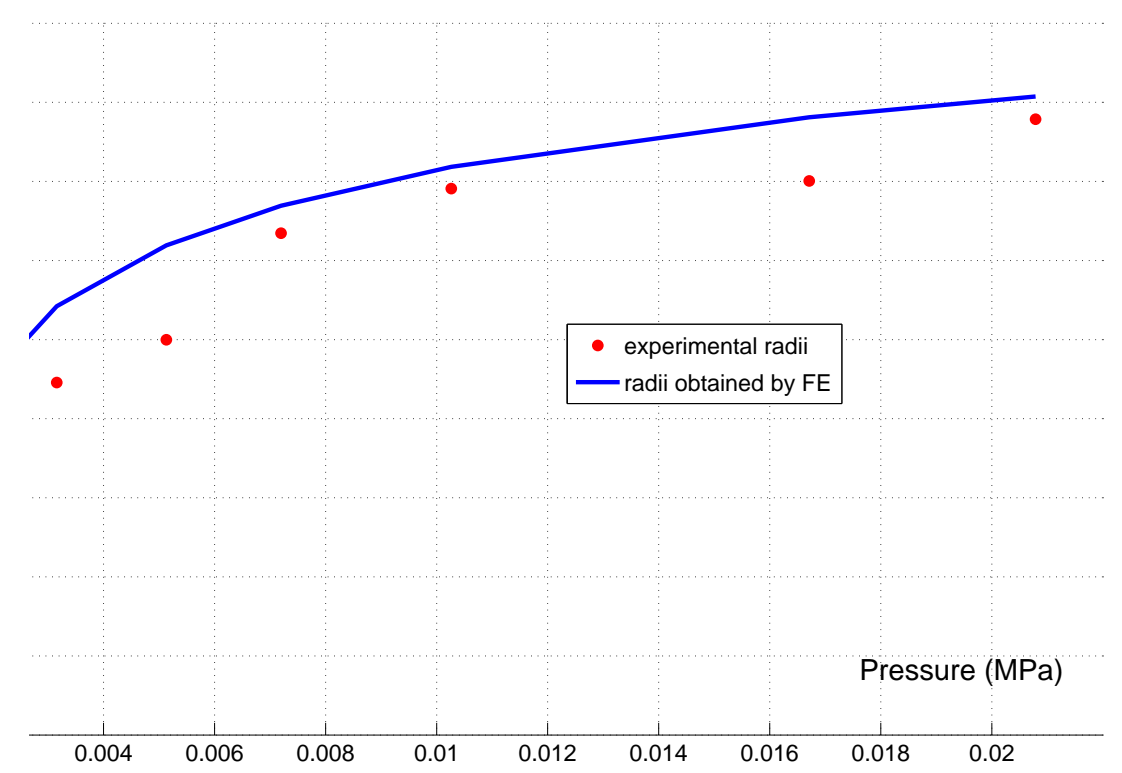

Figure 7: Comparison of the deformed geometry between a FE model and experimental data 
(a)

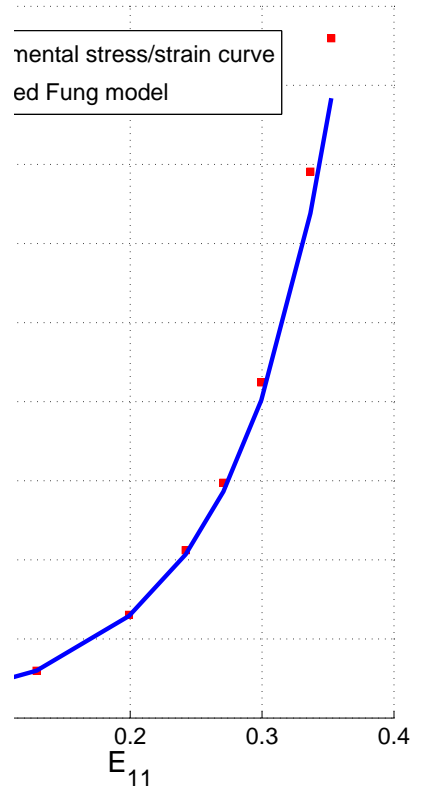

(b)

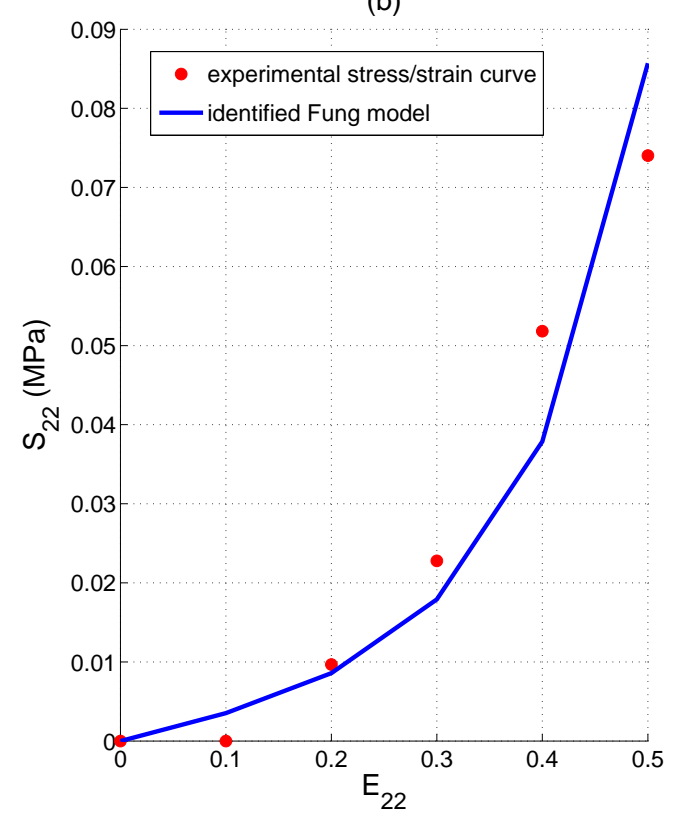

Figure 8: Obtained stress/strain curves: (a) in the circumferential direction and (b) in the axial direction 